\title{
CGA ameliorates cognitive decline by regulating the PI3K/AKT signaling pathway and neurotransmitter systems in rats with multi-infarct dementia
}

\author{
YING FU ${ }^{1,2^{*}}$, JIANGPING WEI ${ }^{1,2^{*}}, \mathrm{BIN} \mathrm{LI}^{2,3^{*}}$, LIJUAN GAO $^{1,2}$, \\ PENG XIA $^{1,2}$, YUEQIANG WEN ${ }^{2,4}$ and SHIJUN XU ${ }^{1,2}$ \\ ${ }^{1}$ School of Pharmacy, Chengdu University of Traditional Chinese Medicine, Chengdu, Sichuan 611137; \\ ${ }^{2}$ Institute of Meterial Medica Integration and Transformation for Brain Disorders, \\ Chengdu University of Traditional Chinese Medicine, Chengdu, Sichuan 611137; \\ ${ }^{3}$ Geriatrics Department, Hospital of Chengdu University of Traditional Chinese Medicine, Chengdu, Sichuan 610075; \\ ${ }^{4}$ School of Basic Medicine, Chengdu University of Traditional Chinese Medicine, Chengdu, Sichuan 611137, P.R. China
}

Received June 5, 2019; Accepted February 13, 2020

DOI: $10.3892 /$ etm. 2020.9198

\begin{abstract}
Multi infarct dementia (MID) is a form of dementia that is preventable and treatable. However, at present, the drugs used in MID treatment were developed for Alzheimer's disease. While only a limited range of drugs is available, the incidence of MID is increasing year on year. The present study aimed to investigate the effect and underlying mechanisms of a combination of ginsenosides and astragalosides (CGA) on cognitive decline in rats with MID. A rat model of MID was established using micro-thromboembolism, and the behavioral changes in the rats were evaluated using the Morris water maze and open field tests at 60 days post-CGA intervention. The pathological morphology of the hippocampal CA1 area was observed using hematoxylin and eosin staining. The contents of ATP, ADP and AMP were determined using high-performance liquid chromatography. Mitochondrial swelling and changes in the membrane potential in the hippocampus were detected using flow cytometry, and the changes in insulin, glutamate and $\gamma$-aminobutyric acid (GABA) content were detected using ELISA. Additionally, the expression of PI3K and AKT proteins was detected using western blot analysis. In a rat model of MID, CGA shortened the escape latency, increased
\end{abstract}

Correspondence to: Mr Yueqiang Wen, School of Basic Medicine, Chengdu University of Traditional Chinese Medicine, 1166 Liutai Avenue, Chengdu, Sichuan 611137, P.R. China

E-mail: wenyueqiang@163.com

Professor Shijun Xu, School of Pharmacy, Chengdu University of Traditional Chinese Medicine, 1166 Liutai Avenue, Chengdu, Sichuan 611137, P.R. China

E-mail: xushijun@cdutcm.edu.cn

*Contributed equally

Key words: astragalosides, energy charge, ginsenosides, multiinfarct dementia, PI3K/AKT signaling pathway the frequency of platform crossing, improved the disordered vertebral cell arrangement and reduced the cell number in the hippocampal CA1 area. CGA also reduced the degree of mitochondrial swelling, increased the mitochondrial membrane potential, and elevated the energy load and ATP content in the brain of rats with MID. Furthermore, CGA increased the insulin content and upregulated the expression of PI3K and $\mathrm{AKT}$ in the brain of rats with MID. In addition, in the rat model of MID, CGA also enhanced the movement time and the frequency of standing, and decreased the concentration of glutamate and GABA in the brain tissue. Amelioration of the cognitive decline in rats with MID by CGA was associated with its regulatory effect on the PI3K/AKT signaling pathway and neurotransmitter systems.

\section{Introduction}

Multi-infarct dementia (MID) is a common type of vascular dementia that is characterized by multiple lesions and infarction of small arteries in the cerebral gray-white matter (1). The brain is an organ that consumes a high amount of energy and requires stable blood flow to deliver a sufficient amount of energy to maintain synaptic activity $(2,3)$. A number of studies have reported that cerebral hypoperfusion is caused by cardiac arrest, arrhythmia and heart failure $(4,5)$, decreased cerebral blood flow induced by hyperlipidemia, atherosclerosis and diabetes (6), as well as ageing and the apolipoprotein E gene (7), which are major risk factors for cognitive impairment. Insufficient or low cerebral blood flow, especially acute cerebral ischemia, can cause insufficient glucose uptake, significantly reduced ATP content (8) and the accumulation of lactic acid in the brain (9). The mitochondria is the main site of ATP synthesis (10) and an excessive accumulation of lactic acid can cause mitochondrial dysfunction (11), inducing oxidative stress, mitochondrial autophagy and apoptosis $(12,13)$, which in turn exacerbate MID lesions and cognitive decline. Therefore, regulating energy metabolism in the brain may be an effective strategy to improve cognitive impairment in MID. 
Saponin, which is the main active constituent of ginseng and astrogalus, has also been reported to significantly improve symptoms in ischemic disease animal models (14-17) and cognitive decline in dementia animal models (18-21). However, the use of a combination of ginseng and astrogalaus (CGA) saponin in vascular dementia has not yet been reported. Therefore, the present study aimed to investigate the effect of CGA on cognitive function in rats with MID and to explore the potential mechanisms of action from the aspect of energy regulation.

\section{Materials and methods}

Animals. A total of 65 male specific pathogen-free Spraque-Dawley rats (weight, $200 \pm 20$ g; age, 6 weeks) were purchased from Chengdu Dashuo Experimental Animal Co., Ltd. All rats were maintained at $22 \pm 2{ }^{\circ} \mathrm{C}$ with $65 \pm 5 \%$ humidity and had free access to drinking water and feed on a 12-h light/dark cycle. The experimental procedure was approved by the Institute of Materia Medica Integration and Transformation for Brain Disorders Ethical Committee.

Drugs and reagents. The ginseng and astrogalus total saponins were purchased from Baoji Herbest Bio-Tech Co., Ltd. (batch nos. 20171120 and 20171121, respectively). CGA is a combination of ginseng total saponin (cat. no. 20171120; purity, 90\% determined by supplier using HPLC.) and astragaloside total saponin (cat. no. 20171121; purity, 90\% determined by supplier using HPLC) at 1:1. ATP, ADP and AMP disodium salt were purchased from Sigma-Aldrich; Merck KGaA.

Model preparation and grouping. After 3 days of adaptive feeding, the MID rat model was prepared using the micro-thromboembolic method, as previously described (24-25). The sham-operation group received the same surgical procedure, but the thrombus was replaced with normal saline. At 2 weeks post-operation, Y maze was used to evaluate the success of modeling. Animals were assigned to treatment groups following the Y-maze task; groups were counterbalanced based on the number of errors animals made during the Y-maze task, such that there was no difference in error times between treatment groups $(\mathrm{P}=0.999$ for error times) The evaluation criteria (26) were as follows: If the number of errors was $>5$, the modeling was deemed successful, animals that were not successful were excluded; successful model rats were ranked from low to high according to the number of errors and then put into five cages in sequence according to a zigzag method (27) to ensure that the number of errors by rats in each cage was not significantly different. The cages were randomly assigned to the following groups ( $\mathrm{n}=10$ per group): Model, Hydergine $(0.7 \mathrm{mg} / \mathrm{kg}$; Tianjin Huajin Pharmaceutical Co., Ltd.), high-dose CGA (CGAH; 20 mg/ml), low-dose CGA (CGAL; $10 \mathrm{mg} / \mathrm{ml})(22,23)$ and control groups. The control group consisted of 10 sham-operated rats. The rats in the drug treatment groups were intragastrically administered with the corresponding drugs once per day for 60 days, at a dose of $10 \mathrm{ml} / \mathrm{kg}$. The rats in the sham-operation and model groups were intragastrically administered with $10 \mathrm{ml} / \mathrm{kg}$ of normal saline once per day for 60 consecutive days.

\section{Morris water maze}

Hidden platform experiment. On day 54 of drug intervention, the five-day hidden platform experiment was performed in all groups of rats. The rats were trained twice a day, during which the time taken to find the platform within $60 \mathrm{sec}$ (escape latency period) was recorded for each rat. If a rat failed to find the platform within $60 \mathrm{sec}$, it was guided to and kept on the platform for $10 \mathrm{sec}$, and the escape latency was recorded as $60 \mathrm{sec}$.

Spatial probe test. The spatial probe test was implemented on day 59 of drug intervention. The platform was removed. Subsequently, the rats were placed into the maze in the same position and their movement trajectories, as well as their frequency of crossing the position that formerly held the platform, within $60 \mathrm{sec}$ were recorded.

Open field test. The open field test was performed with an OFT-100 opening experiment system (TechMan Software, WMT-100; Chengdu Taimeng Software Co., Ltd.). The movement time, immobile period and frequency of standing in a 5 min session were recorded and used as evaluating indicators.

Mitochondrial swelling and membrane potential detection. After the water maze experiment, a total of 24 rats were sacrificed and $80 \mathrm{mg}$ of brain tissue was collected from the hippocampus of each rat. Manual grinding with PBS using a glass homogenizer and divided the homogenate into two parts, then centrifuged $\left(600 \mathrm{x} \mathrm{g} ; 5 \mathrm{~min} ; 4^{\circ} \mathrm{C}\right)$ with one part of the homogenate, and the supernatantcarefully transferred to another tube for further centrifugation $\left(1,200 \mathrm{x} \mathrm{g} ; 10 \mathrm{~min} ; 4^{\circ} \mathrm{C}\right)$. The supernatant was discarded and the precipitate resuspended in $500 \mu$ l Mitochondria Storage Buffer (cat. no. C3609; Beyotime Institute of Biotechnology) at a concentration of $1 \times 10^{5}$ cells $/ \mathrm{ml}$ per tube, mixed for the detection of mitochondrial swelling. The second part of homogenate was collected and the concentration was adjusted to $1 \times 10^{6}$ cells $/ \mathrm{ml}$. Subsequently, $5 \mu \mathrm{l}$ of a mitochondrial membrane potential detection JC-1 kit (cat. no. 551302; Becton-Dickinson and Company) was added to $100 \mu \mathrm{l} \mathrm{sample}$, incubated at $37^{\circ} \mathrm{C}$ for $15 \mathrm{~min}$ and then washed with PBS. After centrifugation $\left(350 \mathrm{x} \mathrm{g} ; 5 \mathrm{~min} ; 4^{\circ} \mathrm{C}\right)$. The precipitate was collected. The precipitate was resuspended in 300-400 $\mu \mathrm{l}$ JC-1 dilution, mixed and mounted for the detection of mitochondrial membrane potential. Mitochondrial swelling and membrane potential were analyzed using a flow cytometer (CytoFLEX; Beckman Coulter, Inc.) and Kaluza software (version 2.1; Beckman Coulter, Inc.)

Detection of hippocampus energy load. A total of $65 \mathrm{mg}$ of hippocampus tissue was collected, mixed with normal saline (weight of hippocampus: Normal saline volume, 1:10) and homogenized in an ice bath. Subsequently, $0.6 \mathrm{ml}$ homogenate was collected, mixed with an equal volume of $0.5 \mathrm{~mol} / \mathrm{l}$ perchloric acid in a vortex mixer, and centrifuged at $4^{\circ} \mathrm{C}$ and $12,000 \times \mathrm{g}$ for $10 \mathrm{~min}$. Furthermore, $0.8 \mathrm{ml}$ supernatant was collected, and the $\mathrm{pH}$ was neutralized using $\mathrm{NaOH}(5 \mathrm{~mol} / \mathrm{l})$. The supernatant was allowed to stand for $30 \mathrm{~min}$ and was then centrifuged at $4^{\circ} \mathrm{C}$ and $12,000 \times \mathrm{g}$ for $10 \mathrm{~min}$. Subsequently, the supernatant was directly sampled and analyzed using high-performance liquid chromatography (Agilent1260; Agilent Techonologies, Inc.) to determine the contents of AMP, ADP and ATP. ATP, ADP and AMP disodium salts were used as internal 
Table I. The pathological grading standard.

Grade Microscopic description

\begin{tabular}{ll}
\hline- & No lesion. \\
0. \\
1. Focal edema of gray and white matter, cells dissolved; \\
2. Focal atrophy of gray and white matter, hyperchromatic nuclei and cytoplasm; \\
3. Focal slight cellular proliferation (mainly gliacyte); \\
4. Slight perivascular edema; \\
5. Slight vascular engorgement and hemorrhage; \\
6. Slight inflammatory cell infiltration; \\
7. Slight gray matter atrophy; \\
8. Slight ependymal cells proliferation. \\
It was graded as '+' when conformed to one of all above, score of which was 1. \\
1. Multifocal edema of gray and white matter, cells dissolved; \\
2. Focal atrophy of gray and white matter, hyperchromatic nuclei and cytoplasm; \\
3. Focal cellular proliferation (mainly gliacyte); \\
4. Medium vascular edema and hemorrhage; \\
5. Medium inflammatory cells infiltration; \\
6. Medium perivascular edema. \\
It was graded as '++' when conformed to one of all above, score of which was 2. \\
1. diffuse edema of gray and white matter, cells dissolved; \\
2. Multifocal atrophy of gray and white matter, hyperchromatic nuclei and cytoplasm; \\
3. Focal mass cellular proliferation (mainly gliacyte); \\
4. Severe vascular edema and hemorrhage; \\
5. Severe inflammatory cell infiltration. \\
It was graded as '+++' when conformed to one of all above, score of which was 3. \\
\hline
\end{tabular}

standards. The conditions used were as follows: Hypersil ${ }^{\mathrm{TM}}$ ODS C18 column (particle size, $5 \mu \mathrm{m}$; 250x4.6 mm²); column temperature, $25^{\circ} \mathrm{C}$; The mobile phase is phosphate buffer and methanol, $0.05 \mathrm{~mol} / 1 \mathrm{PBS}$ ( $\mathrm{pH} 6.5$ ); flow rate, $1.0 \mathrm{ml} / \mathrm{min}$; sample temperature, $10^{\circ} \mathrm{C}$; sampling volume, $10 \mu \mathrm{l}$; and ultraviolet wavelength, $254 \mathrm{~nm}$. The energy load was calculated as follows: $\mathrm{EC}=([\mathrm{ATP}]+0.5[\mathrm{ADP}]) /([\mathrm{ATP}]+[\mathrm{ADP}]+[\mathrm{AMP}])$.

Hematoxylin and eosin staining. The rats were sacrificed and placed in an ice bath to harvest the brain. Part of the left hemisphere was fixed with $4 \%$ paraformaldehyde at room temperature for $24 \mathrm{~h}$, replaced with new $4 \%$ paraformaldehyde, and then fixed for $48 \mathrm{~h}$ at room temperature, dehydrated and embedded with paraffin. Subsequently, the tissue was cut into $5 \mu \mathrm{m}$ slices, stained with hematoxylin for $30 \mathrm{~min}$ and eosin for $5 \mathrm{~min}$ at room temperature, vitrified with xylene, sealed with neutral resin, and then placed under a optical microscope (x200; model, Olympus Optical CX22; Olympus Corporation) to observe the morphology of neurons in the hippocampal CA1 region in 3 visual fields of each sample. Pathological scoring was performed as previously described (28), the pathological scoring standard is shown in Table I.

Western blot analysis. Thirty milligrams of brain tissue was weighed, mixed with RIPA buffer (cat. no. WB020; $1 \mathrm{mg}$ : $10 \mu \mathrm{l}$; Multisciences (Lianke) Biotech, Co., Ltd.) homogenized in an ice bath and centrifuged at $4^{\circ} \mathrm{C}$ and $12,000 \mathrm{xg}$ for $10 \mathrm{~min}$.
The resulting supernatant was collected for protein determination by BCA. Equal amounts $(50 \mu \mathrm{g})$ of the total protein were separated by $8 \%$ SDS-PAGE, transferred to a PVDF membrane and blocked with 5\% BSA (cat. no. EZ3416D317; BioFroxx; neoFroxx $\mathrm{GmbH}$ ) at room temperature for $90 \mathrm{~min}$. Subsequently, the membrane was incubated with the following primary antibodies at $4^{\circ} \mathrm{C}$ overnight: anti-PI3K (cat. no. 4257S; 1:1,000; Cell Signaling Technology, Inc.), anti-phosphorylated (p)-PI3K (cat. no. 4228S; 1:1,000; Cell Signaling Technology, Inc.), anti-AKT (cat. no. 4685S; 1:1,000; Cell Signaling Technology, Inc.), anti-p-AKT (cat. no. 4060S; 1:1,000; Cell Signaling Technology, Inc.) and GAPDH (cat. no. 180411; 1:5,000; Wuhan Servicebio Technology Co.,Ltd.). Subsequently, the membrane was washed with TBST solution three times and incubated with the horseradish peroxidase-conjugated goat anti-rabbit IgG secondary antibody [cat. no. A8040064; 1:5,000; Hangzhou Multisciences (Lianke) Biotech Co., Ltd.] at room temperature for $90 \mathrm{~min}$. The membrane was washed with TBST solution three times. Protein bands were visualized using a hypersensitive ECL kit (cat. no. 4AW011-100; Beijing 4A Biotech Co., Ltd.). Protein expression was quantified using Quantity One (version 4.6.2; Bio-Rad Laboratories, Inc.) software with GAPDH as the loading control.

ELISA. A total of $50 \mathrm{mg}$ of brain tissue was weighed and mixed with PBS containing 1\% PMSF (1 mg: $9 \mu \mathrm{l}$ ), homogenized in an ice bath and centrifuged at $4{ }^{\circ} \mathrm{C}$ and $5,000 \mathrm{x} \mathrm{g}$ for $10 \mathrm{~min}$. 

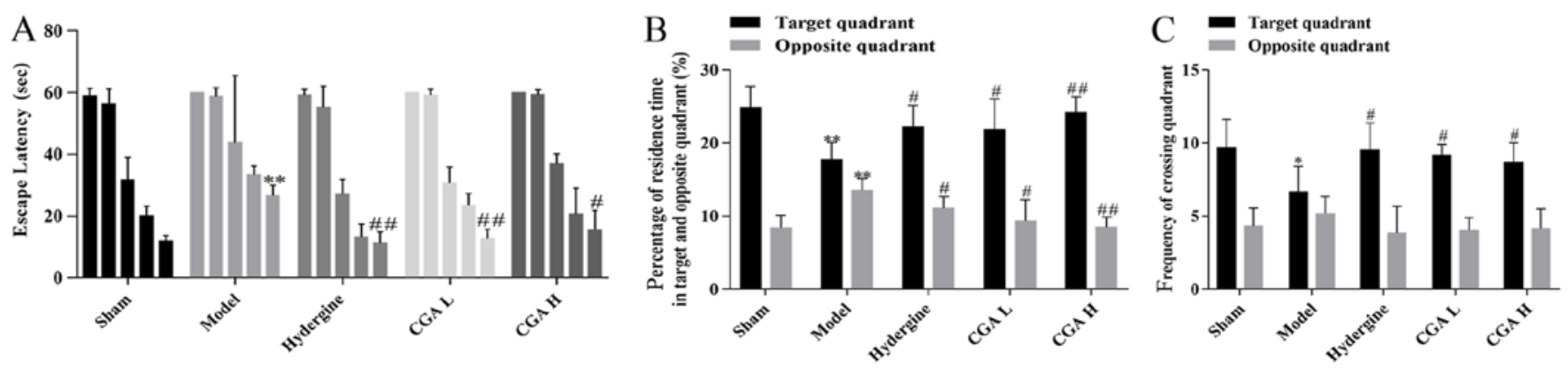

Figure 1. Effect of CGA on learning and memory in rats with MID. (A) Escape latency. (B) Percentage of residence time in target and opposite quadrants. (C) Frequency of crossing target and opposite quadrants. ${ }^{*} \mathrm{P}<0.05$ and ${ }^{* *} \mathrm{P}<0.01$ vs. the sham group; ${ }^{*} \mathrm{P}<0.05$ and ${ }^{\# \#} \mathrm{P}<0.01$ vs. the model group. CGA, compatibility of ginsenosides and astragalosides; MID, multi-infarct dementia; CGA H, high-dose CGA; CGA L, low-dose CGA.

Sham

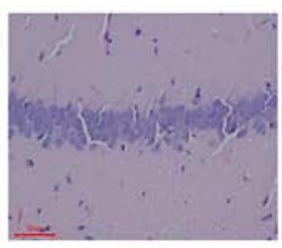

Model

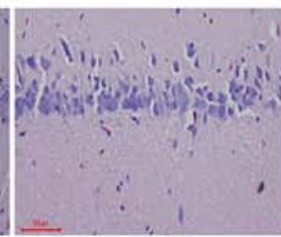

Hydergine

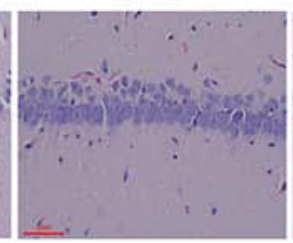

CGA L

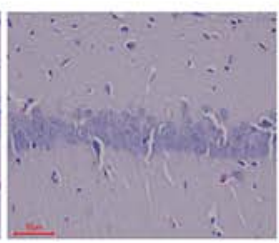

CGAH

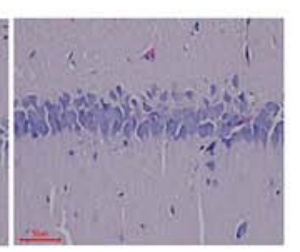

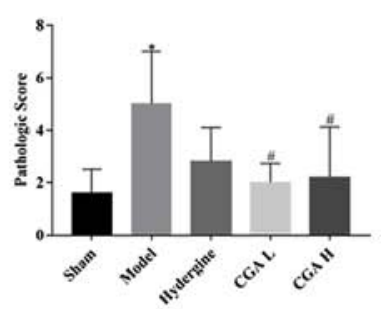

Figure 2. Effect of CGA on the pathomorphology of the hippocampal CA1 region of the brain in rats with $\mathrm{MID}$. ${ }^{*} \mathrm{P}<0.05$ vs. the sham group; ${ }^{*} \mathrm{P}<0.05$ vs. the model group. CGA, compatibility of ginsenosides and astragalosides; MID, multi-infarct dementia; CGA H, high-dose CGA; CGA L, low-dose CGA.

The supernatant was collected. Subsequently, $100 \mu \mathrm{l}$ supernatant was used to determine the concentrations of insulin (Rat insulin ELISA kit; cat. no. E-EL-R2466c ; Elabscience Biotechnology Co., Ltd.), glutamate (glutamic acid measurement kit; cat. no. A074-1-1; Nanjing Jiancheng Bioengineering Institute.) and $\gamma$-aminobutyric acid (GABA) $(\gamma$-aminobutyric acid assay kit; cat. no. H168; Nanjing Jiancheng Bioengineering Institute.), according to the manufacturers' protocols.

Statistical analysis. Data are presented as the mean \pm standard deviation. Statistical analyses of the data was performed in a double-blinded fashion. Morris water maze escape latency data were analyzed using a two-way ANOVA followed by Tukey's post-hoc test. The other data in line with normal distribution were analyzed using a one-way ANOVA followed by Tukey's post-hoc test . The pathological results were analyzed using Kruskal Wallis followed by Dunn's post hoc test. $\mathrm{P}<0.05$ was considered to indicate a statistically significant difference.

\section{Results}

Effect of CGA on learning and memory in rats with MID. The escape latency of each group displayed a time-dependent pattern, and was indicated to decrease as training time increased (Fig. 1A). The escape latency of the rats was significantly increased in the model group compared with the sham group, while the escape latency of the rats in the CGA group was significantly shorter than the model groups (Fig. 1A). Meanwhile, the percentage of residence time in the target quadrant in the model group was decreased compared with the sham, hydergine and CGA groups, but the percentage in the opposite quadrant was increased compared with the sham and CGA H groups (Fig. 1B). Similarly, the frequency of crossing the target quadrant in the model group was significantly reduced compared with the sham, hydergine and CGA groups, while the frequency of crossing the opposite quadrant displayed no statistical difference between all the groups (Fig. 1C).

Effect of CGA on the pathomorphology of hippocampal CA1 area in rats with MID. The vertebral nerve cells in the hippocampal CA1 area were sparsely arranged, and the number of cells with incomplete structure, nerve cell necrosis and degeneration was increased in the model group compared with the sham group $(\mathrm{P}<0.05$; Fig. 2). The sparse arrangement, necrosis and degeneration of the nerve cells were significantly improved in the CGA groups compared with the model group ( $\mathrm{P}<0.05$; Fig. 2).

Effect of CGA on hippocampal mitochondrial function in rats with $M I D$. The mitochondrial membrane potential in the hippocampus decreased significantly in the model group compared with the sham group $(\mathrm{P}<0.01$; Fig. $3 \mathrm{~A}$ and $\mathrm{B})$. The CGA groups displayed significantly reversed effects of MID on the mitochondrial membrane potential compared with the model group $(\mathrm{P}<0.05$; Fig. $3 \mathrm{~A}$ and $\mathrm{B})$. Furthermore, the mitochondrial swelling in the hippocampus was significantly increased in the model group compared with the sham group $(\mathrm{P}<0.01$; Fig. $3 \mathrm{C}$ and $\mathrm{D})$, while the mitochondrial swelling was significantly decreased in the CGA H and CGA L groups compared with the model group $(\mathrm{P}<0.05$; Fig. $3 \mathrm{C}$ and $\mathrm{D})$.

Effect of CGA on brain energy load in rats with MID. ATP content in the brain tissue was significantly decreased ( $\mathrm{P}<0.05$; Fig. 4A), AMP and ADP contents were not significantly altered ( $\mathrm{P}>0.05$; Fig. $4 \mathrm{~B}$ and $\mathrm{C})$, and the brain energy load was significantly decreased $(\mathrm{P}<0.05$; Fig. $4 \mathrm{D})$ in the model 

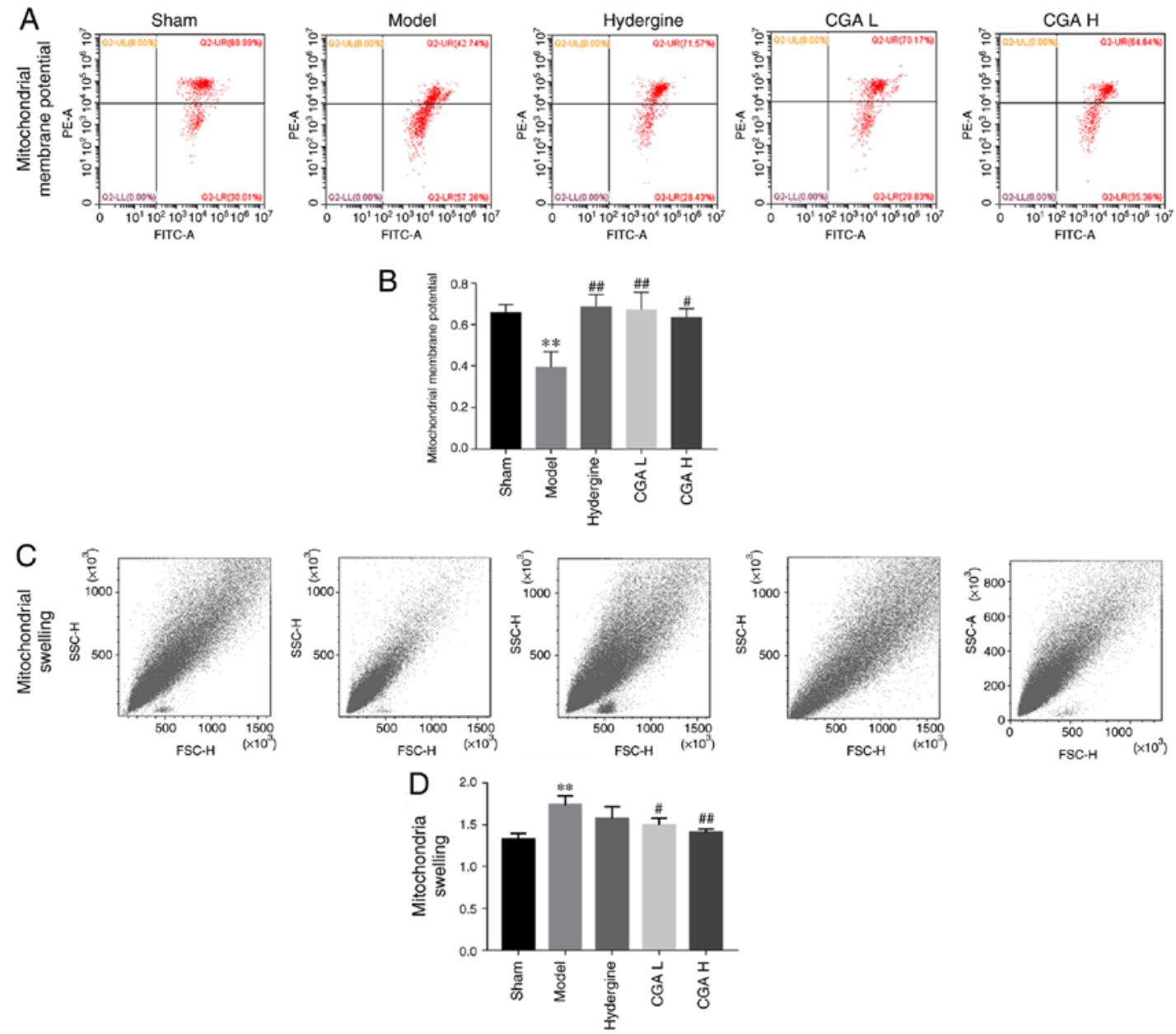

Figure 3. Effect of CGA on hippocampal mitochondrial function in rats with MID. Flow cytometry (A) analysis and (B) quantification of mitochondrial membrane potential. Flow cytometry $(\mathrm{C})$ analysis and (D) quantification of mitrochondrial swelling. ${ }^{* *} \mathrm{P}<0.01$ vs. the sham group; ${ }^{\#} \mathrm{P}<0.05$ and ${ }^{\# \#} \mathrm{P}<0.01$ vs. the model group CGA, compatibility of ginsenosides and astragalosides; MID, multi-infarct dementia; CGA H, high-dose CGA; CGA L, low-dose CGA; PE-A, phycoerythrin-A; SSC-H, side scatter-height; FSC-H, forward scatter-height.

group compared with the sham group. The ATP content in the brain tissue was significantly increased $(\mathrm{P}<0.05 ;$ Fig. $4 \mathrm{~A})$, AMP and ADP contents were not significantly altered $(\mathrm{P}>0.05$; Fig. 4B and C), and the brain energy load was significantly increased $(\mathrm{P}<0.05$; Fig. 4D) in the CGA and Hydergine groups compared with the model group.

Effect of CGA on the PI3K/AKT signaling pathway in rats with $M I D$. The insulin content in the brain was significantly lower in the model group compared with the sham group $(\mathrm{P}<0.05$; Fig. 5A). However, the insulin content in the brain was significantly increased in the CGA and Hydergine groups compared with the model group $(\mathrm{P}<0.05$; Fig. 5A). The ratio of $\mathrm{p}-\mathrm{PI} 3 \mathrm{~K} / \mathrm{PI} 3 \mathrm{~K}$ and $\mathrm{p}-\mathrm{AKT} / \mathrm{AKT}$ in the brain was significantly decreased in the model group compared with the sham group (Fig. $5 \mathrm{~B}$ and $\mathrm{C}$ ). In contrast, the ratio of $\mathrm{p}-\mathrm{PI} 3 \mathrm{~K} / \mathrm{PI} 3 \mathrm{~K}$ and $\mathrm{p}-\mathrm{AKT} / \mathrm{AKT}$ was significantly increased in the CGA and Hydergine groups compared with the model group (Fig. 5B and C).

Effect of CGA on the concentration of neurotransmitters. A number of emotional behavioral changes were observed in the model rats, except cognitive decline. The movement time was significantly lower in the model group compared with the sham group $(\mathrm{P}<0.05$; Fig. 6A). Furthermore, the silent period was significantly increased in the model group compared with the sham group $(\mathrm{P}<0.05$; Fig. $6 \mathrm{~B})$. However, the effect of the MID model on both movement time and immobile period were reversed by administering CGA H. Meanwhile, the MID model-induced lower frequency of standing was also reversed by the administration of CGA ( $\mathrm{P}<0.05$; Fig. 6C). Subsequently, the concentration of GABA and glutamate in the brain tissues was detected. The concentration of glutamate in the brain tissue of model rats was higher compared with the sham rats $(\mathrm{P}<0.05$; Fig. 6D). When compared with the model group, the concentration of glutamate in the Hydergine and CGA groups decreased significantly ( $\mathrm{P}<0.05$; Fig. 6D). CGA also significantly reduced the GABA content in the brain of MID model rats when compared with the model group $(\mathrm{P}<0.01$; Fig. $6 \mathrm{E})$.

\section{Discussion}

Worldwide, vascular dementia is the second most common form of dementia accompanied by obvious cognitive dysfunction after Alzheimer's disease (29), and MID is a common type of vascular dementia. Currently, no licensed drugs are available for the treatment of vascular dementia (30), and the reported therapeutic strategies for the disease primarily 

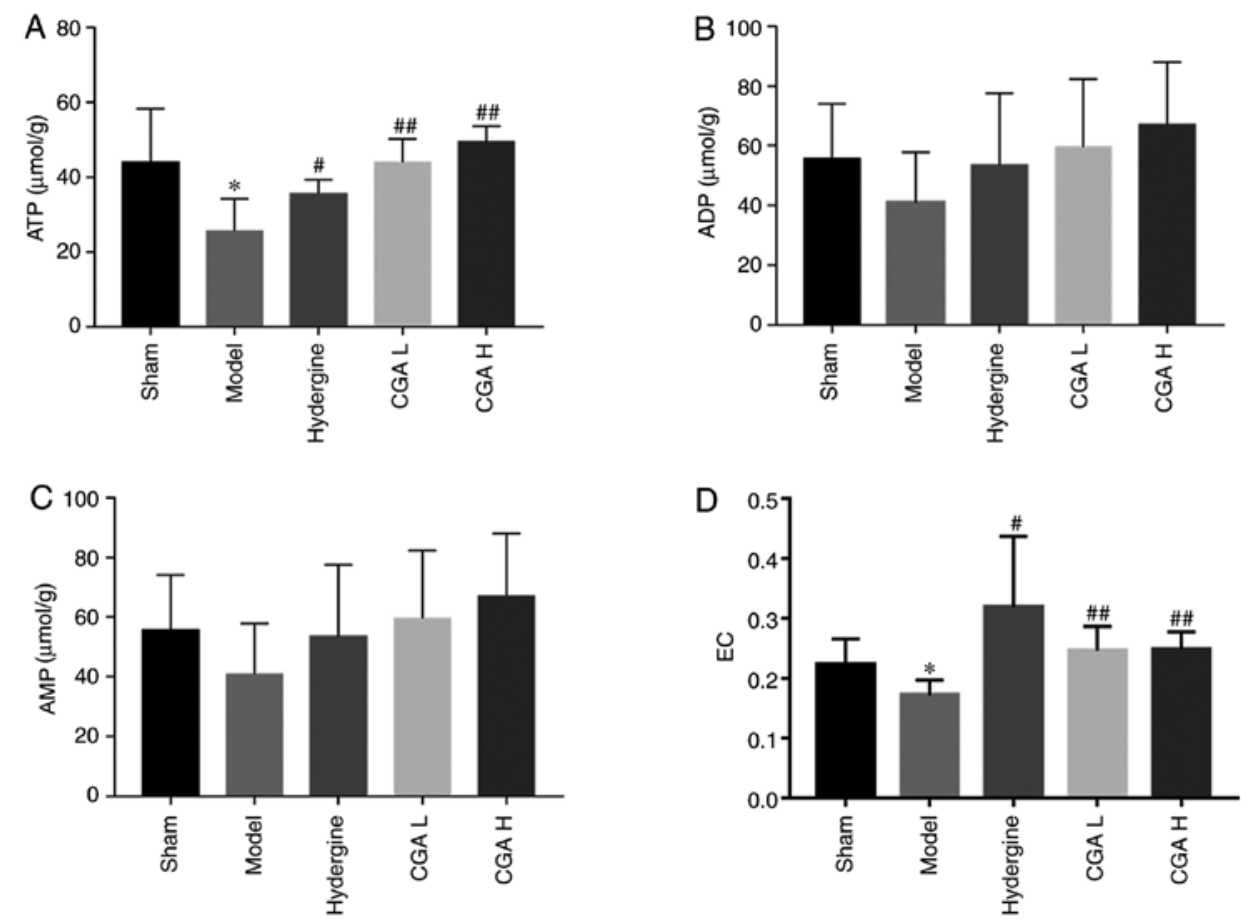

Figure 4. Effect of CGA on brain energy load in rats with MID. (A) ATP, (B) ADP and (C) AMP content. (D) EC. "P $<0.05$ vs. the sham group; "P $<0.05$ and ${ }^{\# \#} \mathrm{P}<0.01$ vs. the model group. CGA, compatibility of ginsenosides and astragalosides; MID, multi-infarct dementia; CGA H, high-dose CGA; CGA L, low-dose CGA; EC, energy charge.
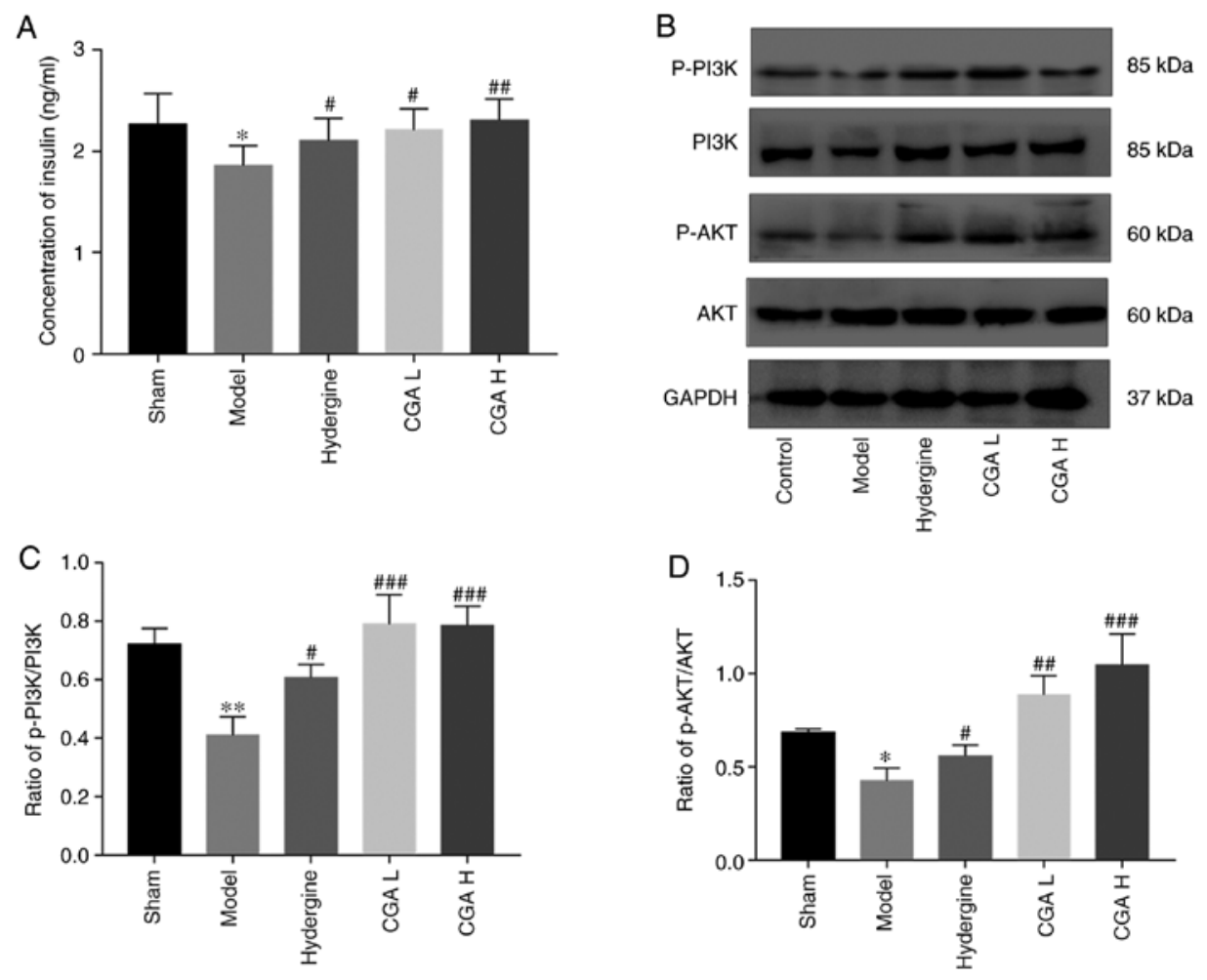

Figure 5. Effect of CGA on the insulin content and PI3K/AKT protein expression levels in the hippocampus of rats with MID. (A) Concentration of insulin. Levels of p-PI3K, PI3K, p-AKT and AKT protein expression were (B) determined by western blot analysis and the ratio of (C) p-PI3K/PI3K and (D) p-AKT/AKT were quantified. ${ }^{*} \mathrm{P}<0.05$ and ${ }^{* * *} \mathrm{P}<0.01$ vs. the sham group; ${ }^{*} \mathrm{P}<0.05$, ${ }^{\# \#} \mathrm{P}<0.01$ and ${ }^{\# \# \#} \mathrm{P}<0.001$ vs. the model group. CGA, compatibility of ginsenosides and astragalosides; MID, multi-infarct dementia; p, phosphorylated; CGA H, high-dose CGA; CGA L, low-dose CGA.

focus on inhibiting oxidative stress, apoptosis and inflammation $(31,32)$. Multiple and cortical microinfarctions are closely related to dementia and cognition (33), which is associated with their direct influence on the production of ATP in the brain (7). Therefore, regulating the energy metabolism in the brain and maintaining the physiological function of normal nerve cells 

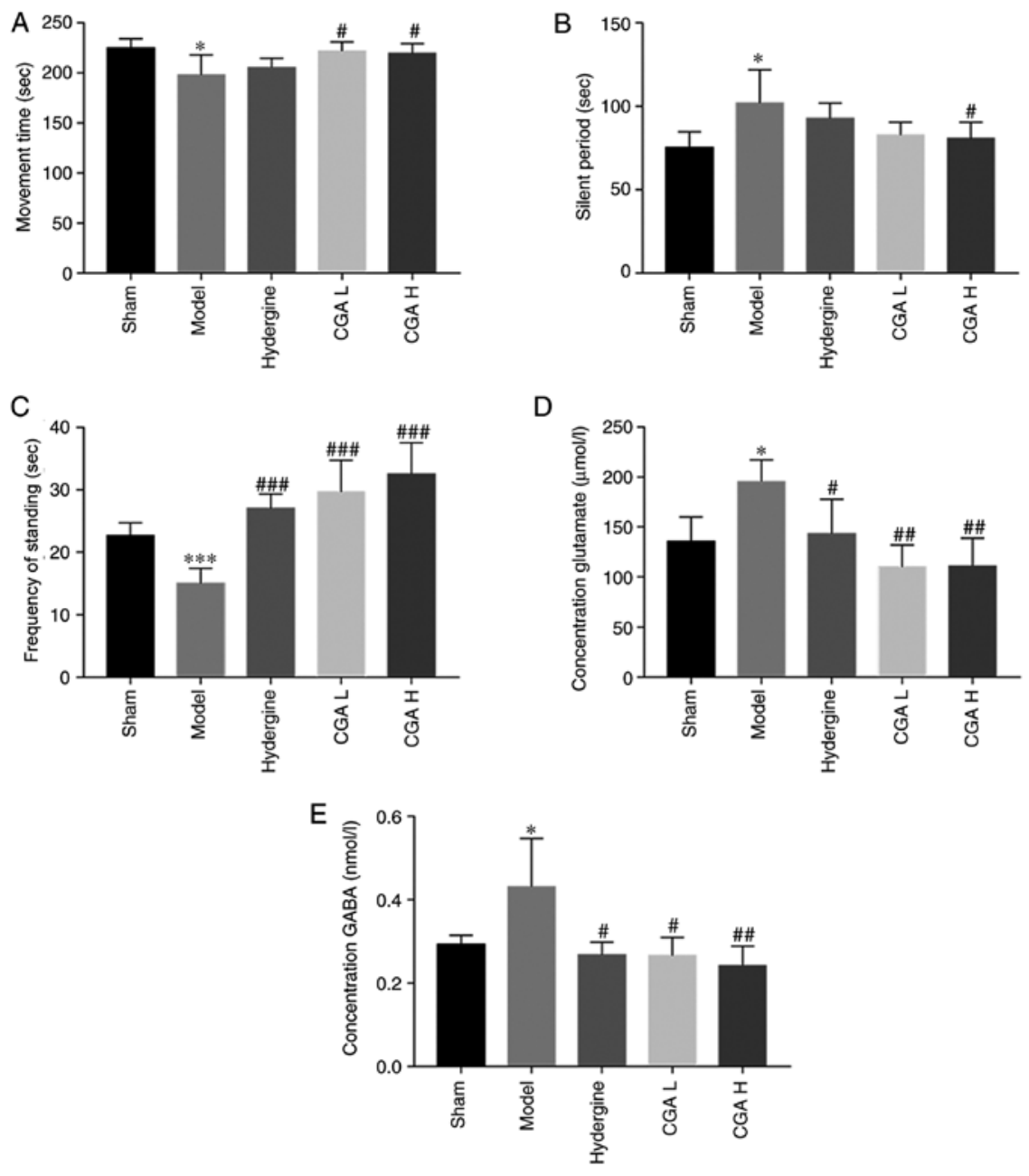

Figure 6. Effect of CGA on the behavior and neurotransmitter systems of rats with MID. The (A) movement time, (B) silent period and (C) frequency of standing of rats, measured by an open field test. Concentration of (D) glutamate and (E) GABA. ${ }^{*} \mathrm{P}<0.05$ and ${ }^{* * * *} \mathrm{P}<0.01$ vs. the sham group. ${ }^{\# P} \mathrm{P}<0.05$, ${ }^{\# \prime} \mathrm{P}<0.01$ and ${ }^{\# \# \#} \mathrm{P}<0.001$ vs. the model group. CGA, compatibility of ginsenosides and astragalosides; MID, multi-infarct dementia; GABA, $\gamma$-aminobutyric acid; CGA H, high-dose CGA; CGA L, low-dose CGA.

in patients with MID may be effective therapeutic strategies in the treatment of this disease. The present study suggested that CGA significantly improved the cognitive function, and disordered arrangement and sparseness of vertebral cells in the hippocampal CA1 area in rats with MID. Furthermore, CGA regulated mitochondrial swelling and membrane potential, and significantly increased ATP content and brain energy load in rats with MID. In addition, CGA significantly increased the insulin content and the expression of PI3K and AKT in the brain of rats with MID.

The presence of multiple arteriolar vascular infarctions in the gray matter is a key characteristic of MID (34). Injection of a small amount of thrombus (diameter, 50-100 $\mu \mathrm{m}$ ) into the internal carotid artery can simulate multiple arterial infarctions in cognitive areas, including the cortex and hippocampus (35), making it an ideal method for modeling MID. The present study displayed significantly declined cognitive function, lesions in the hippocampal CA1 area, structural and functional impairment of the mitochondria, and brain ATP and energy load in rats with MID. Continuous low-level brain energy not only affects neuronal function, but also induces autophagy and apoptosis via the mTOR, PI3K, peroxisome proliferator activated receptor- $\gamma$ and AMP-activated protein kinase signaling pathways (36).
The PI3K/AKT signaling pathway is a key regulator involved in the metabolism, growth, proliferation and survival of cells, which can be activated by multiple receptors or proteins, including insulin, insulin-like growth factor-1, low-density lipoprotein-related receptor 1 and toll-like receptors (37-39). After being activated by insulin, the PI3K signaling pathway is involved primarily in energy metabolism in vivo. The present study suggested that the insulin content and expression of PI3K and AKT proteins was significantly decreased in the brain of rats with MID compared with control rats.

Saponin is the main active substance of ginseng and Huangqi, and has been reported to improve learning and memory functions in a number of studies $(14,15,17,20)$. The present study suggested that CGA significantly improved the decline in the learning and memory of rats with MID and improved the degeneration and necrosis of nerve cells in the hippocampal CA1 area. A number of studies have reported that the active ingredients of ginseng, including $\mathrm{Rb} 1, \mathrm{Rg} 1, \mathrm{Rh} 2 \mathrm{E} 2$, $\mathrm{Re}, \mathrm{Rg} 3, \mathrm{Rd}$ and $\mathrm{Rf}$, can regulate energy metabolism (40-46). Similarly, the saponin ingredient of Huangqi exerts a similar effect to Astragaloside IV, which regulates energy metabolism by regulating glycolytic pathways $(47,48)$. The present study suggested that CGA regulated the structure and function of the 
mitochondria, increased brain ATP content and energy load, and significantly increased insulin content in the brain of rats with MID. It further revealed that CGA significantly upregulated the phosphorylation levels of PI3K and AKT, therefore, CGA may activate the PI3K/AKT signaling pathway to regulate energy metabolism. However, identifying the specific saponin ingredients that activate the suppressed PI3K/AKT signaling pathway in MID requires further investigation.

Glutamate and GABA are excitatory and inhibitory neurotransmitters, respectively (49). A large number of studies have indicated a role for both neurotransmitters in the occurrence and development of dementia, similar to other neurotransmitters, including acetylcholine (50-52). Similarly, glutamic acid and GABA in the nerves located in the ventral tegmental region display a regulating effect on risk factors for dementia, including insomnia and insufficient sleep (53). The present study suggested that the activity and standing times of MID model animals decreased significantly, and the contents of glutamate and GABA in the brain tissue increased abnormally compared with control rats, which was similar to the results reported in the aforementioned studies. GABA is derived primarily from glutamate metabolism, indicating why the two neurotransmitters should display an opposite pattern of expression (54). The relationship between GABA and glutamate in the present study was consistent with previous findings (55-57), suggesting that either the source of glutamate increased or the elimination of GABA decreased. However, further investigation is required to clarify this.

In conclusion, improvements in brain energy metabolism in rats with MID by CGA was closely associated with the regulatory effect of CGA on the PI3K/AKT signaling pathway and neurotransmitter systems. The majority of drugs that have been reported to improve dementia display antioxidant, anti-inflammatory and antiapoptotic mechanisms. However, the present study focused on energy metabolism and to the best of our knowledge, suggested for the first time that CGA regulated energy metabolism via the PI3K/AKT signaling pathway to achieve an anti-vascular dementia effect. The results of the present study suggested that the use of CGA may be beneficial for the treatment of vascular dementia.

\section{Acknowledgements}

Not applicable.

\section{Funding}

The present study was supported by the Key Research and Development Plan of Sichuan Province (grant no. 19ZDYF0600), the program of Traditional Chinese Medicine Bureau of Sichuan Province (grant no. 2018JC013), the Program of Education Department of Sichuan Province (grant no. 17ZA0149), the Program of Chengdu University of Traditional Chinese Medicine Science and Technology Development Fund (grant no. ZRYY1727).

\section{Availability of data and materials}

The datasets used and/or analyzed during the current study are available from the corresponding author on reasonable request.

\section{Authors' contributions}

SX conceived, designed the study and critically reviewed, edited, and revised the paper; YW conceived and revised the final manuscript. YF performed the experiments and drafted the original manuscript. JW performed the experiments, data analysis and revised the manuscript. BL guided the partial experimental methods and critically reviewed and revised the original manuscript. All authors read and approved the final manuscript.

\section{Ethics approval and consent to participate}

The experimental procedure was approved by the Institute of Meterial Medica Integration and Transformation for Brain Disorders Ethical Committee.

\section{Patient consent for publication}

Not applicable.

\section{Competing interests}

The authors declare that they have no competing interests.

\section{References}

1. Iadecola C: The pathobiology of vascular dementia. Neuron 80: 844-866, 2013.

2. Iadecola C: Neurovascular regulation in the normal brain and in Alzheimer's disease. Nat Rev Neurosci 5: 347-360, 2004.

3. Harris JJ, Jolivet R and Attwell D: Synaptic energy use and supply. Neuron 75: 762-777, 2012.

4. Stefansdottir H, Arnar DO, Aspelund T, Sigurdsson S, Jonsdottir MK, Hjaltason H, Launer LJ and Gudnason V: Atrial fibrillation is associated with reduced brain volume and cognitive function independent of cerebral infarcts. Stroke 44: 1020-1025, 2013.

5. Marshall RS: Effects of altered cerebral hemodynamics on cognitive function. J Alzheimers Dis 32: 633-642, 2012.

6. de la Torre JC: Cerebral hemodynamics and vascular risk factors: Setting the stage for Alzheimer's disease. J Alzheimers Dis 32: 553-567, 2012.

7. Qian J, Wolters FJ, Beiser A, Haan M, Ikram MA, Karlawish J, Langbaum JB, Neuhaus JM, Reiman EM, Roberts JS, et al: APOE-related risk of mild cognitive impairment and dementia for prevention trials: An analysis of four cohorts. PLoS Med 14: e1002254, 2017.

8. Tian J, Fu F, Geng M, Jiang Y, Yang J, Jiang W, Wang C and Liu K: Neuroprotective effect of $20(\mathrm{~S})$-ginsenoside $\mathrm{Rg} 3$ on cerebral ischemia in rats. Neurosci Lett 374: 92-97, 2005.

9. Feng Y, Zhang W and Guo J: The alterations of brain lactate, lactate dehydrogenase, creatine phosphokinase and its influence on these of peripheral blood or liver tissue and entero-barrier during brain hypoperfusion. Zhongguo Bing Li Sheng Li Xue Hui 12: 1106-1109, 1999 (In Chinese).

10. van der Bliek AM, Sedensky MM and Morgan PG: Cell Biology of the Mitochondrion. Genetics 207: 843-871, 2017.

11. Parihar MS and Brewer GJ: Amyloid- $\beta$ as a modulator of synaptic plasticity. J Alzheimers Dis 22: 741-763, 2010.

12. Li H, Liu Y, Lin LT, Wang XR, Du SQ, Yan CQ, He T, Yang JW and Liu CZ: Acupuncture reversed hippocampal mitochondrial dysfunction in vascular dementia rats. Neurochem Int 92: 35-42, 2016.

13. Park YS, Choi SE and Koh HC: PGAM5 regulates PINK1/Parkin-mediated mitophagy via DRP1 in CCCP-induced mitochondrial dysfunction. Toxicol Lett 284: 120-128, 2018.

14. Wan Q, Ma X, Zhang ZJ, Sun T, Xia F, Zhao G and Wu YM: Ginsenoside reduces cognitive impairment during chronic cerebral hypoperfusion through brain-derived neurotrophic factor regulated by epigenetic modulation. Mol Neurobiol 54: 2889-2900, 2017. 
15. 1Dong $\mathrm{X}$, Zheng L, Lu S and Yang Y: Neuroprotective effects of pretreatment of ginsenoside Rb1 on severe cerebral ischemia-induced injuries in aged mice: Involvement of anti-oxidant signaling. Geriatr Gerontol Int 17: 338-345, 2017.

16. Tang B, Wang D, Li M, Wu Q, Yang Q, Shi W and Chen C: An in vivo study of hypoxia-inducible factor-1 $\alpha$ signaling in ginsenoside Rg1-mediated brain repair after hypoxia/ischemia brain injury. Pediatr Res 81: 120-126, 2017.

17. Li M, Li H, Fang F, Deng X and Ma S: Astragaloside IV attenuates cognitive impairments induced by transient cerebral ischemia and reperfusion in mice via anti-inflammatory mechanisms. Neurosci Lett 639: 114-119, 2017.

18. Zong W, Zeng X, Chen S, Chen L, Zhou L, Wang X, Gao Q, Zeng $\mathrm{G}, \mathrm{Hu} \mathrm{K}$ and Ouyang D: Ginsenoside compound $\mathrm{K}$ attenuates cognitive deficits in vascular dementia rats by reducing the A $\beta$ deposition. J Pharmacol Sci 139: 223-230, 2019.

19. Zhang G, Liu A, Zhou Y, San X, Jin T and Jin Y: Panax ginseng ginsenoside-Rg2 protects memory impairment via anti-apoptosis in a rat model with vascular dementia. J Ethnopharmacol 115 441-448, 2008

20. Chang CP, Liu YF, Lin HJ, Hsu CC, Cheng BC, Liu WP, Lin MT, Hsu SF, Chang LS and Lin KC: Beneficial effect of astragaloside on Alzheimer's disease condition using cultured primary cortical cells under $\beta$-amyloid exposure. Mol Neurobiol 53: 7329-7340, 2016.

21. Li WZ, Wu WY, Huang DK, Yin YY, Kan HW, Wang X, Yao YY and Li WP: Protective effects of astragalosides on dexamethasone and A $325-35$ induced learning and memory impairments due to decrease amyloid precursor protein expression in 12-month male rats. Food Chem Toxicol 50: 1883-1890, 2012.

22. Zhang Y, Su H, Zhang J and Kong J: The effects of ginsenosides and anserine on the up-regulation of renal aquaporins 1-4 in hyperuricemic mice. Am J Chin Med 47: 1133-1147, 2019.

23. Qiu LH, Zhang BQ, Lian MJ, Xie XJ and Chen P: Vascular protective effects of and its main constituents in rats with chronic hyperhomocysteinemia. Exp Ther Med 14: 2401-2407, 2017.

24. Yang JW, Wang XR, Ma SM, Yang NN, Li QQ and Liu CZ: Acupuncture attenuates cognitive impairment, oxidative stress and $\mathrm{NF}-\kappa \mathrm{B}$ activation in cerebral multi-infarct rats. Acupunct Med 37: 283-291, 2019.

25. Zhang X, Wu B, Nie K, Jia Y and Yu J: Effects of acupuncture on declined cerebral blood flow, impaired mitochondrial respiratory function and oxidative stress in multi-infarct dementia rats Neurochem Int 65: 23-29, 2014.

26. Ying S, Lai X, Guan C, Xie LL, Wu LN and Tang CZ: Effect of electroacupuncture on learning-memory ability of vascular dementia rats with concomitant hypertension and hyperlipemia. Zhen Ci Yan Jiu 34: 368-375, 2009 (In Chinese).

27. Lin C, Jiang F, Peng T, et al: Toxicity test of zsydo on rabbit embryo fetal development//2015 (fifth) annual meeting of pharmacotoxicology.

28. Ren X, Wei J and Gong D: Tongluoxingnao effervescent tablets ameliorate learning and memory impairment in a rat model of vascular dementia via the regulation of the p38 and ERK MAPK signaling pathways. Int J Clin Exp Med 9: 5400-5412, 2016.

29. Smith EE: Clinical presentations and epidemiology of vascular dementia. Clin Sci (Lond) 131: 1059-1068, 2017.

30. O'Brien JT and Thomas A: Vascular dementia. Lancet 386: $1698-1706,2015$

31. Gubandru M, Margina D, Tsitsimpikou C, Goutzourelas N, Tsarouhas K, Ilie M, Tsatsakis AM and Kouretas D: Alzheimer's disease treated patients showed different patterns for oxidative stress and inflammation markers. Food Chem Toxicol 61: 209-214, 2013.

32. BagheriG,RezaeeR,TsarouhasK,Docea AO,ShahrakiJ,ShahriariM, Wilks MF, Jahantigh H, Tabrizian K, Moghadam AA, et al Magnesium sulfate ameliorates carbon monoxide induced cerebral injury in male rats. Mol Med Rep 19: 1032-1039, 2019.

33. Arvanitakis Z, Leurgans SE, Barnes LL, Bennett DA and Schneider JA: Microinfarct pathology, dementia, and cognitive systems. Stroke 42: 722-727, 2011.

34. Ferrari C, Nacmias B and Sorbi S: The diagnosis of dementias: A practical tool not to miss rare causes. Neurol Sci 39: 615-627, 2018

35. Kaneko D, Nakamura $\mathrm{N}$ and Ogawa T: Cerebral infarction in rats using homologous blood emboli: Development of a new experimental model. Stroke 16: 76-84, 1985.

36. Hou K, Xu D, Li F, Chen S and Li Y: The progress of neuronal autophagy in cerebral ischemia stroke: Mechanisms, roles and research methods. J Neurol Sci 400: 72-82, 2019.

37. Yang L, Wang H,Liu L and Xie A: The role of insulin/IGF-1/PI3K/ Akt/GSK3 $\beta$ signaling in Parkinson's disease dementia. Front Neurosci 12: 73, 2018
38. Peng J, Pang J, Huang L, Enkhjargal B, Zhang T, Mo J, Wu P, $\mathrm{Xu}$ W, Zuo Y, Peng J, et al: LRP1 activation attenuates white matter injury by modulating microglial polarization through Shc1/PI3K/Akt pathway after subarachnoid hemorrhage in rats. Redox Biol 21: 101121, 2019.

39. Lv Y, Liu W, Ruan Z, Xu Z and Fu L: Myosin IIA regulated tight junction in oxygen glucose-deprived brain endothelial cells via activation of TLR4/PI3K/Akt/JNK1/2/14-3-3e/NF-kB/MMP9 signal transduction pathway. Cell Mol Neurobiol 39: 301-319, 2019.

40. Li L, Pan CS, Yan L, Cui YC, Liu YY, Mu HN, He K, Hu BH, Chang X, Sun K, et al: Ginsenoside Rg1 ameliorates rat myocardial ischemia-reperfusion injury by modulating energy metabolism pathways. Front Physiol 9: 78, 2018.

41. Zhou P, Xie W, He S, Sun Y, Meng X, Sun G and Sun X: Ginsenoside $\mathrm{Rb} 1$ as an anti-diabetic agent and its underlying mechanism analysis. Cells 8: 204, 2019.

42. Wong VK, Dong H, Liang X, Bai LP, Jiang ZH, Guo Y, Kong AN, Wang R, Kam RK, Law BY, et al: Rh2E2, a novel metabolic suppressor, specifically inhibits energy-based metabolism of tumor cells. Oncotarget 7: 9907-9924, 2016.

43. Nam Y,Wie MB,Shin E, Nguyen TL,Nah S, Ko SK, Jeong JH,Jang C and Kim H: Ginsenoside Re protects methamphetamine-induced mitochondrial burdens and proapoptosis via genetic inhibition of protein kinase $\mathrm{C} \delta$ in human neuroblastoma dopaminergic SH-SY5Y cell lines. J Appl Toxicol 35: 927-944, 2015.

44. Li J, Liu T, Zhao L, Chen W, Hou H, Ye Z and Li X: Ginsenoside 20(S) Rg3 inhibits the Warburg effect through STAT3 pathways in ovarian cancer cells. Int J Oncol 46: 775-781, 2015.

45. Zhou JS, Wang JF, He BR, Cui YS, Fang XY, Ni JL, Chen J and Wang KZ: Ginsenoside Rd attenuates mitochondrial permeability transition and cytochrome $\mathrm{C}$ release in isolated spinal cord mitochondria: Involvement of kinase-mediated pathways. Int J Mol Sci 15: 9859-9877, 2014.

46. Shangguan WJ,LiH and Zhang YH: Induction of G2/M phase cell cycle arrest and apoptosis by ginsenoside Rf in human osteosarcoma MG 63 cells through the mitochondrial pathway. Oncol Rep 31: 305-313, 2014.

47. Zhang C, Cai T, Zeng X, Cai D, Chen Y, Huang X, Gan H, Zhuo J, Zhao Z, Pan H, et al: Astragaloside IV reverses MNNG-induced precancerous lesions of gastric carcinoma in rats: Regulation on glycolysis through miRNA-34a/LDHA pathway. Phytother Res 32: 1364-1372, 2018.

48. Dong Z, Zhao P, Xu M, Zhang C, Guo W, Chen H, Tian J, Wei H, Lu R and Cao T: Astragaloside IV alleviates heart failure via activating PPAR $\alpha$ to switch glycolysis to fatty acid $\beta$-oxidation. Sci Rep 7: 2691, 2017.

49. Pereira AC, Mao X, Jiang CS, Kang G, Milrad S, McEwen BS, Krieger AC and Shungu DC: Dorsolateral prefrontal cortex GABA deficit in older adults with sleep-disordered breathing. Proc Natl Acad Sci USA 114: 10250-10255, 2017.

50. Kwakowsky A, Calvo-Flores Guzmán B, Pandya M, Turner C, Waldvogel HJ and Faull RL: GABAA receptor subunit expression changes in the human Alzheimer's disease hippocampus, subiculum, entorhinal cortex and superior temporal gyrus. J Neurochem 145: 374-392, 2018.

51. Jo S, Yarishkin O, Hwang YJ, Chun YE, Park M, Woo DH, Bae JY, Kim T, Lee J, Chun H, et al: GABA from reactive astrocytes impairs memory in mouse models of Alzheimer's disease. Nat Med 20: 886-896, 2014.

52. Mayor D and Tymianski M: Neurotransmitters in the mediation of cerebral ischemic injury. Neuropharmacology 134: 178-188, 2018.

53. Yu X, Li W, Ma Y, Tossell K, Harris JJ,Harding EC, Ba W, Miracca G, Wang D, Li L, et al: GABA and glutamate neurons in the VTA regulate sleep and wakefulness. Nat Neurosci 22: 106-119, 2019.

54. Petroff OA: GABA and glutamate in the human brain. Neuroscientist 8: 562-573, 2002.

55. Long H, Ruan J, Zhang M, Wang $\mathrm{C}$ and Huang Y: Gastrodin alleviates Tourette syndrome via Nrf-2/HO-1/HMGB1/NF-KB pathway. J Biochem Mol Toxicol 33: e22389, 2019.

56. Jiang B, Meng L, Zou N, Wang H, Li S, Huang L, Cheng X, Wang Z, Chen W and Wang C: Mechanism-based pharmacokinetics-pharmacodynamics studies of harmine and harmaline on neurotransmitters regulatory effects in healthy rats: Challenge on monoamine oxidase and acetylcholinesterase inhibition. Phytomedicine 62: 152967, 2019.

57. Liu J, Wu Y-Y, Yu X-L, Jia HY, Mao QY and Fang JQ: Temporal effect of acupuncture on amino acid neurotransmitters in rats with acute cerebral ischaemia. Acupunct Med 37: 252-258, 2019.

This work is licensed under a Creative Commons Attribution-NonCommercial-NoDerivatives 4.0 International (CC BY-NC-ND 4.0) License. 